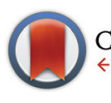

CrossMark $\leftarrow$ click for updates

Cite this: Org. Biomol. Chem., 2017, 15, 301

Received 26th October 2016, Accepted 23rd November 2016

DOI: $10.1039 / c 6 o b 02333 a$

www.rsc.org/obc

\section{Diastereoselective synthesis of trifluoromethylated 1,3-dioxanes by intramolecular oxa-Michael reaction $\dagger$}

Liliana Becerra-Figueroa, ${ }^{a}$ Elodie Brun, ${ }^{\mathrm{b}}$ Michael Mathieson, ${ }^{\mathrm{b}}$ Louis J. Farrugia, ${ }^{\mathrm{b}}$ Claire Wilson, ${ }^{\mathrm{b}}$ Joëlle Prunet ${ }^{\star b}$ and Diego Gamba-Sánchez ${ }^{\star a}$

\begin{abstract}
A highly diastereoselective synthesis of trifluoromethylated 1,3dioxanes is described. The reaction proceeds by an addition/oxaMichael sequence and works efficiently under mild reaction conditions, with a good substrate scope and acceptable to good yields.
\end{abstract}

Trifluoromethyl-containing heterocycles and particularly oxygenated heterocyclic systems have attracted growing attention in the last few years. ${ }^{1}$ They are currently investigated because of their interesting applications in medicine, agriculture and materials science, among others. ${ }^{2}$ In spite of the continuous growth in this field, the synthesis of 1,3-dioxanes remains unexplored. Consequently, the development of new and efficient synthetic methods for this kind of heterocyclic system is at the same time a challenge and very desirable.

Since the seminal work published by Evans and Prunet in $1993,{ }^{3}$ the addition/oxa-Michael cascade has demonstrated to be useful in the construction of protected syn 1,3-diols ${ }^{4}$ (Scheme 1). The reaction substrates are homoallylic alcohols functionalised with a Michael acceptor. The most usual reagent is benzaldehyde but there are several examples in the literature where $p$-methoxy benzaldehyde has been used successfully. ${ }^{5}$ Concerning the Michael acceptor and the substitution pattern the reaction has been explored with esters and amides, ${ }^{3}$ sulfones ${ }^{6}$ and sulfoxides, ${ }^{7}$ and in very few cases with substituents $\alpha$ to the Michael acceptor. ${ }^{8}$

Inspired by those previous results and by the need for a simple method to synthesise $\mathrm{CF}_{3}$-containing dioxanes, we decided to explore the intramolecular oxa-Michael reaction

\footnotetext{
${ }^{a}$ Laboratory of Organic Synthesis, Bio and Organocatalysis, Chemistry Department, Universidad de los Andes, Cra 1 No. 18A-12 Q:305, Bogotá 111711, Colombia. E-mail: joelle.prunet@glasgow.ac.uk, da.gamba1361@uniandes.edu.co

${ }^{b}$ WestCHEM, School of Chemistry, University of Glasgow, Joseph Black Building, University Avenue, Glasgow G12 8QQ, UK

$\dagger$ Electronic supplementary information (ESI) available: Crystallographic data; experimental details and copies of NMR spectra. CCDC 1510756 and 1511482. For ESI and crystallographic data in CIF or other electronic format see DOI: 10.1039/c6ob02333a
}

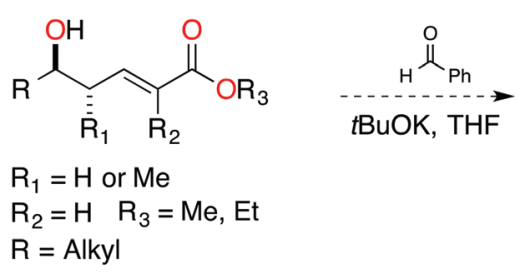<smiles>[R5]C(=O)C([R2])[C@H]1OC(c2ccccc2)OC([R])[C@@H]1[R]</smiles>

Previous approach

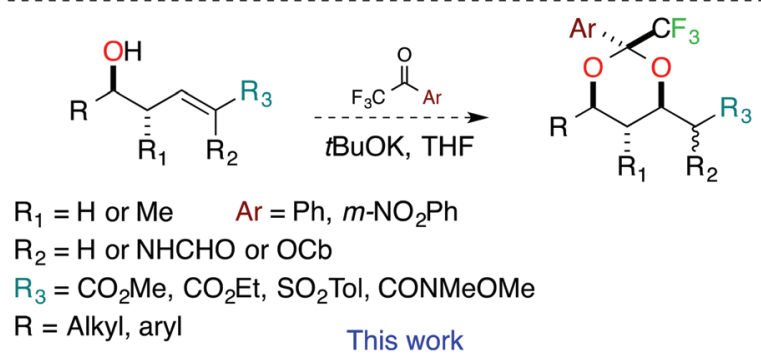

Scheme 1 Difference between the closest similar report and our work.

using trifluoroacetophenone and a homoallylic alcohol functionalised with an ester as substrates (Scheme 1).

During our studies, a similar transformation on cyclic dienones in the presence of triethylamine was described by Wang et al. ${ }^{9}$ Even if the yields were good, the selectivity was moderate in most cases.

With our substrates, if the reaction is under thermodynamic control as it is the case with benzaldehyde, ${ }^{3}$ the major diastereomer should have all the large substituents at equatorial positions (the two alkyl chains and presumably the trifluoromethyl group).

We started our investigation with the simple known ester $1 a^{10}$ and explored the reaction under the conditions described by Wang et al. (Table 1, entry 1). After 24 hours no reaction was observed, and the starting material was recovered quantitatively. The use of a larger quantity of a base and ketone or the use of another weak base such as potassium acetate did not afford better results (entries 2 and 3); therefore, we 
Table 1 Optimisation of the reaction conditions

\begin{tabular}{llllll}
\hline & & & & \\
& & & & & \\
\hline
\end{tabular}

${ }^{a}$ The reaction was performed making successive additions of the base and the ketone every $15 \mathrm{~min} .{ }^{b}$ Determined by ${ }^{1} \mathrm{H}$ NMR of the crude product. ${ }^{c} 80 \%$ brsm.

decided to turn our attention to bases and reaction conditions more familiar to us. ${ }^{11}$

With $t$ BuOLi and 2 equivalents of ketone (entry 4 ), the reaction proceeded and the desired dioxane $2 \mathrm{a}$ was isolated in $49 \%$ yield with a moderate selectivity, the major product has the same stereochemistry in all cases (see below). The change of the counterion to potassium provided much better results and in 6 hours the product was isolated in 84\% yield with good selectivity (entry 5). Typically an intramolecular conjugate addition reaction of this kind is performed using successive addition of a base and carbonyl compound; it was demonstrated that this serves to improve the selectivity and to increase the reaction yield. ${ }^{3}$ Hence, we used three additions of 1.1 equivalents of ketone and 0.1 equivalents of base, but after 2 hours the reaction was not complete and the product was only isolated in $70 \%$ yield ( $80 \%$ brsm, entry 6$)$; however after 6 hours the starting material was completely consumed and the product was isolated in $79 \%$ yield (entry 7 ). In both cases only one diastereomer was detected in the ${ }^{1} \mathrm{H}$ NMR spectrum of the crude reaction mixture and after purification of the product.

The stereochemistry of the major diastereomer remained uncertain. We were confident about the cis stereochemistry of the substituents at positions 4 and 6, but for position 2 the configuration will be dependent on the relative steric hindrance of $\mathrm{Ph}$ and $\mathrm{CF}_{3}$. Fortunately, compound 2a was crystalline and X-ray crystallographic analysis provided its structure (Fig. 1), showing that $\mathrm{CF}_{3}$ is indeed at the equatorial position.

With these promising results in hand, we started the study of the reaction scope. All the starting materials $\mathbf{1}$ were obtained by a cross metathesis reaction between the corresponding homoallylic alcohol and methyl acrylate using the Grubbs II catalyst $(2.5 \% \mathrm{~mol})$. These homoallylic alcohols were easily prepared in one step from commercially available aldehydes and allyl bromide using an aqueous Barbier reaction. ${ }^{12}$ Then we applied the optimised conditions to all substrates $\mathbf{1}$ and we found excellent diastereoselectivity in all cases (see Table 2).

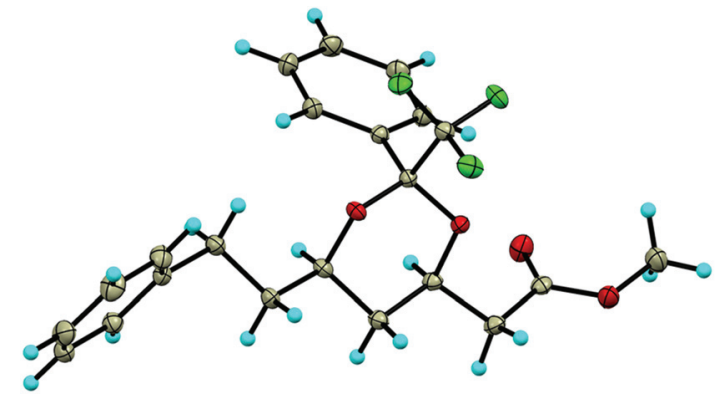

Fig. 1 X-ray structure of product $2 \mathrm{a}$.

Table 2 Reaction scope ${ }^{a}$

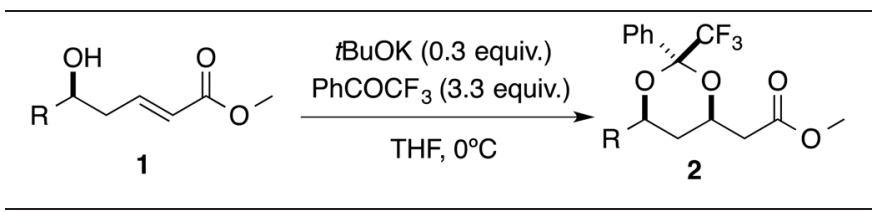

\begin{tabular}{llll}
\hline Entry & $\mathrm{R}$ & Product & Yield $^{b}(\%)$ \\
\hline 1 & $\mathrm{PhCH}_{2} \mathrm{CH}_{2}$ & $\mathbf{2 a}$ & 79 \\
2 & $\mathrm{C}_{6} \mathrm{H}_{13}$ & $\mathbf{2 b}$ & 80 \\
3 & Isopropyl & $\mathbf{2 c}$ & 81 \\
4 & $\mathrm{Chx}$ & $\mathbf{2 d}$ & 82 \\
5 & $\mathrm{CH}_{3} \mathrm{CHOBn}^{c}$ & $\mathbf{2 e}$ & 70 \\
6 & $\mathrm{Ph}_{7}$ & $\mathbf{2 f}$ & $67^{d}$ \\
7 & $p-\mathrm{MeOPh}$ & $\mathbf{2 g}$ & 70 \\
8 & $p-\mathrm{ClPh}$ & $\mathbf{2 h}$ & 63
\end{tabular}

${ }^{a}$ The reaction was performed making successive additions of the base ( 0.1 equiv.) and the ketone (1.1 equiv.) every $15 \mathrm{~min} .{ }^{b}$ Yields are reported for pure compounds; the ${ }^{1} \mathrm{H}$ NMR spectra of the crude reaction mixtures showed in all cases only one diastereomer. ${ }^{c}$ This product was obtained from enantiopure lactic acid and the anti diastereomer from the cross metathesis reaction was isolated (see the ESI). ${ }^{d} 80 \%$ brsm.

The use of simple aliphatic substrates afforded the best results (entries 1 to 4 ); there is no noticeable effect induced by the chain extension, or by the presence of aromatic rings in the aliphatic chain (compare entries 1 and 2). The use of branched $\mathrm{R}$ groups is also acceptable and there is no influence on the reaction diastereoselectivity; in fact, as these groups are at the equatorial position we indeed expected better selectivities with these bulkier $\mathrm{R}$ groups. The reaction is also useful with aromatic $\mathrm{R}$ substituents (entries 6 to 8); however, yields are somehow lower when electron-withdrawing groups are inserted (entry $8 v s$. entry 7 ).

The reaction mechanism is shown in Scheme 2. We postulated that this reaction is under thermodynamic control as it is the case with benzaldehyde, ${ }^{3}$ with a reversible oxa-Michael reaction. So, it is to be expected that the use of a more electrophilic carbonyl compound (trifluoroacetophenone) will cause a better reaction with the alkoxide 3 , favouring the formation of hemiketal alkoxide 4. We hypothesise that this intermediate is stabilised by the inductive effect of the $\mathrm{CF}_{3}$ group, facilitating the reverse oxa-Michael addition and in consequence improving the selectivity of the whole process. 


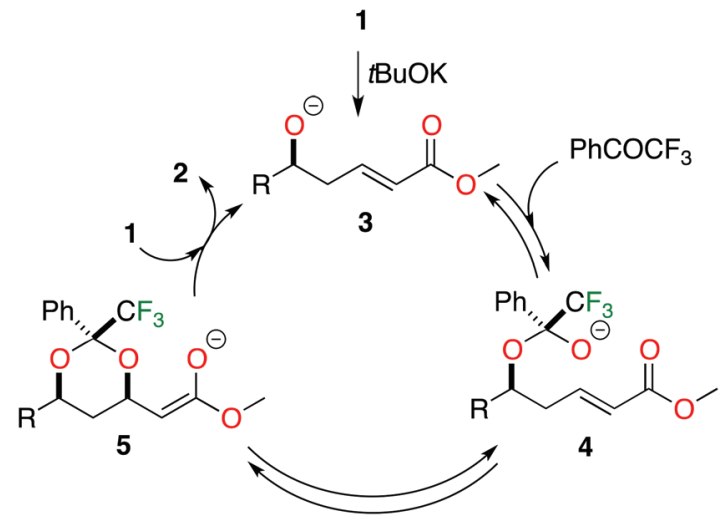

Scheme 2 Reaction mechanism.

Table 3 Reaction with $\alpha$-substituted substrates

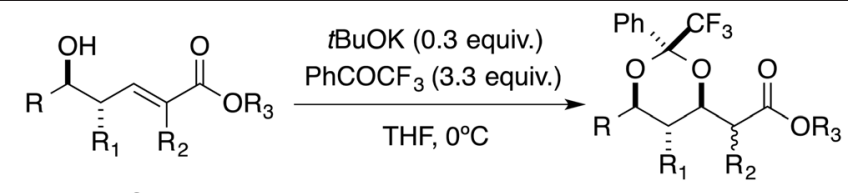

7

\begin{tabular}{llllllc}
\hline Entry & $\mathrm{R}$ & $\mathrm{R}_{1}$ & $\mathrm{R}_{2}$ & $\mathrm{R}_{3}$ & Product, yield & Selectivity \\
\hline 1 & $\mathrm{PhCH}_{2} \mathrm{CH}_{2}$ & $\mathrm{H}$ & $\mathrm{Me}$ & $\mathrm{Et}$ & $7 \mathbf{a},<27 \%^{a}$ & $82: 18$ \\
2 & $\mathrm{PhCH}_{2} \mathrm{CH}_{2}$ & $\mathrm{H}$ & $\mathrm{OCb}^{b}$ & Et & $7 \mathbf{b}, 54 \%^{c}$ & $>98: 2$ \\
$3^{d}$ & $\mathrm{PhCH}_{2} \mathrm{CH}_{2}$ & $\mathrm{Me}$ & $\mathrm{OCb}$ & Et & $7 \mathbf{c}, 90 \%$ & $>98: 2$ \\
$4^{e}$ & $\mathrm{PhCH}_{2} \mathrm{CH}_{2}$ & $\mathrm{H}$ & NHCHO & Me & $7 \mathbf{d}, 85 \%$ & $82: 18$
\end{tabular}

${ }^{a}$ This value was calculated by analysis of the ${ }^{1} \mathrm{H}$ NMR spectrum; the crude mixture also contained the intermediate hemiketal and the elimination product; a sample was purified for analytical purpose. ${ }^{b} \mathrm{Cb}=$ $\mathrm{CON}(\mathrm{iPr})_{2} \cdot{ }^{c} 83 \% \mathrm{brsm} .{ }^{d}$ The reaction was performed using 4 additions of a base and ketone. ${ }^{e}$ The starting material was treated with 1.1 equivalents of base and ketone, and then consecutive additions of 0.1 equivalents of base and 1.1 equivalents of ketones were made.

The next step was the investigation of the effect of a substituent at the $\alpha$ position to the electron-withdrawing group ${ }^{8}$ (results in Table 3); for this purpose, we synthesised several $\alpha$-substituted substrates 6 (see the $\mathrm{ESI}_{\dagger} \dagger$ for the reaction sequence). When the substituent at the $\alpha$ position was a methyl group (entry 1), the outcome of the reaction was disappointing. The product was observed in the ${ }^{1} \mathrm{H}$ NMR spectrum along with the intermediate hemiketal and the elimination product. We were able to isolate the desired ketal with acceptable selectivity but in low yield. On the other hand, the reaction with product $\mathbf{6 b}$ works smoothly and the product was isolated with excellent selectivity and in acceptable yield (entry 2 ); curiously the reaction was not complete after 12 hours and some starting material was recovered. The introduction of a methyl group $\gamma$ to the ester did not have a significant effect on the selectivity (entry 3); in this case the reaction was pushed to completion with 4 additions of a base and ketone to furnish $7 \mathbf{c}$ in $90 \%$ yield. It has to be noted that the reaction on a substrate similar to $\mathbf{6 c}$ (with $\mathrm{R}=\mathrm{iPr}$ ) with benzaldehyde as the electrophilic reagent furnished the corresponding product in $35 \%$ yield (53\% brsm) with a $67: 33$ selectivity. ${ }^{8 d}$

We were also curious about the reactivity of a substrate with a nitrogen substituent at the $\alpha$ position. Depending on the substitution pattern of the nitrogen atom, the compound can have an enamine character or the $\mathrm{N}-\mathrm{H}$ group can be acidic enough to be deprotonated before the alcohol.

We were able to synthesize compound $\mathbf{6 d}$ by a HornerWadsworth-Emmons reaction. ${ }^{13}$ As expected an extra equivalent of base was needed in order to completely deprotonate the NH group (entry 4). It is remarkable that this reaction proceeded giving very good yield and good selectivity, even if the deprotonation of the nitrogen can induce a nucleophilic character to the carbon at the $\beta$ position to the ester. It is worth noting that in all the cases described in Table 3, the reported selectivity corresponds to the position of $\mathrm{R}_{2}$; the reaction proceeds with complete selectivity for the oxygen addition.

Fortunately, the stereochemistry of the substituent at the $\alpha$ position could be determined by X-ray crystallographic analysis of compound $\mathbf{7 d}$ as shown in Fig. 2. Comparison of NMR signals and coupling constants for the $\mathrm{H}-5$ proton in $7 \mathbf{d}$ and 7b allowed us to define the configuration at C5 for compound 7b as shown in Scheme 3. The stereochemistry at C5 of 7c $\mathbf{M}$ was assumed to be the same as that of $\mathbf{7 b}$.

Some additional experiments were carried out in order to illustrate the generality of our method. First, other Michael

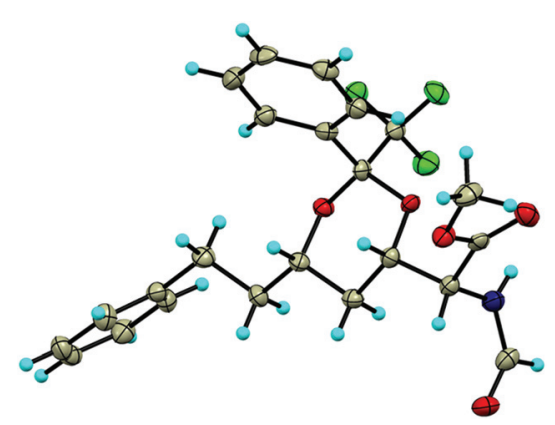

Fig. 2 X-ray structure of product 7c.<smiles>COC(=O)[C@H](NC=O)[C@H]1CC(CCc2ccccc2)OC(c2ccccc2)(c2ccccc2)O1</smiles>

7d M, $J_{4-5}=2.1 \mathrm{~Hz}$ Structure determined by X-Ray<smiles>CCOC(=O)[C@H](O[C@@H]1CC(CCc2ccccc2)OC(c2ccccc2)(c2ccccc2)O1)C(F)(F)F</smiles>

$7 \mathrm{~b} \mathrm{M}, J_{4-5}=3.3 \mathrm{~Hz}$<smiles>COC(=O)[C@H](NC=O)[C@H]1CC(CCc2ccccc2)OC(c2ccccc2)(c2ccccc2)O1</smiles>

7d m, $J_{4-5}=3.1 \mathrm{~Hz}$
Scheme 3 Stereochemistry for major $(M)$ and minor $(\mathrm{m})$ diastereomers. 
a)

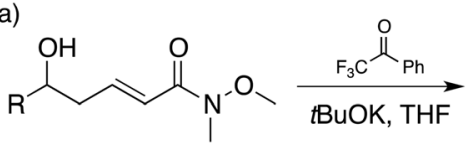

8, $\mathrm{R}=\mathrm{PhCH}_{2} \mathrm{CH}_{2}$

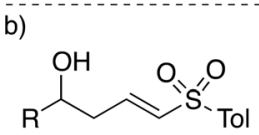

10, $\mathrm{R}=\mathrm{PhCH}_{2} \mathrm{CH}_{2}$

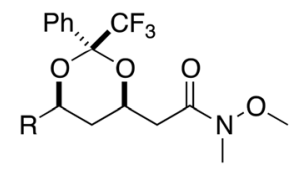

9, $80 \%,(d . r>98: 2)$

Scheme 4 Weinreb amide and sulfone as Michael acceptors.<smiles>[R]C(O)C/C=C/C(=O)OC</smiles>

12, $\mathrm{R}=p-\mathrm{MeOPh}$

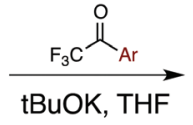

13

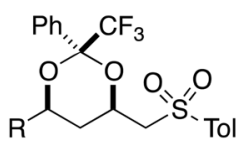

$11,76 \%$, (d. $r>98: 2)$

Scheme 5 Reaction using $m$-nitrotrifluoroacetophenone.

acceptors such as Weinreb amides or sulfones were explored. The cited literature ${ }^{3-8}$ shows that the reactivity of the Michael acceptors does not have a great influence on the yield of the oxa-Michael reaction. The main difference is the diastereoselectivity.

In consequence, we were curious to see if in our case Weinreb amides or sulfones are desirable substrates and if the selectivity is comparable to that obtained with esters.

As shown in Scheme 4 the results are very good, the reaction yields are comparable to those obtained with simple esters and in both cases, Scheme $4 \mathrm{a}$ and $\mathrm{b}$, there was only one diastereoisomer visible by ${ }^{1} \mathrm{H}$ NMR of the crude reaction mixture and isolated products 9 and 11. The stereochemistry of these compounds was assigned by comparison with that of the esters.

We also decided to use another trifluoromethyl ketone; we speculated that, if the ketone was more electrophilic than trifluoroacetophenone, the hemiketal alkoxide $\mathbf{4}$ would be less nucleophilic and so the reaction would be more difficult. Thus, if the reaction worked with a ketone substituted in the aromatic ring by a strong electron withdrawing group, then it would be predictable than the reaction could be extended to the use of almost any trifluoromethyl ketone. Scheme 5 shows the result of the reaction using $m$-nitro-trifluoroacetophenone as the electrophilic reagent. In this case the reaction proved to be more difficult; four additions of a base and 16 hours were needed to complete the reaction; however, product 13 was isolated in good yield and the reaction proceeded with complete diastereoselectivity.

In summary, the additional experiments allow us to conclude that different Michael acceptors and other trifluoromethyl ketones can be used, thus making the method very general.

Finally, computational studies oriented to propose an explanation for the difference in reactivity and selectivity between the substrates, as well as additional experiments to increase even more the reaction scope are currently under investigation in our laboratory. The results will be reported in due course.

In conclusion, we have described a useful methodology for the synthesis of $\mathrm{CF}_{3}$-containing 1,3-dioxanes with excellent diastereoselectivity in most cases. We also reported our preliminary conclusions about the reaction mechanism based on literature reports and experimental observations. The stereochemistry of the products was established by X-ray crystallographic analysis and extrapolation.

Financial support for this work was provided by the University of Glasgow, the EPSRC (Doctoral Training Allocation EP/P50418X/1 for M. M.) and Universidad de los Andes. L. B.-F acknowledges the Universidad de los Andes and especially the Chemistry Department for a fellowship and the Faculty of Science for financial support for her internship. We also thank Mr David Sotelo for recording some of the NMR spectra.

\section{Notes and references}

1 (a) F. M. D. Ismail, J. Fluorine Chem., 2002, 118, 27; (b) J.-A. Ma and D. Cahard, Chem. Rev., 2004, 104, 6119; (c) J. Nie, H.-C. Guo, D. Cahard and J.-A. Ma, Chem. Rev., 2011, 111, 455; (d) Y.-J. Lin, L.-N. Du, T.-R. Kang, Q.-Z. Liu, Z.-Q. Chen and L. He, Chem. - Eur. J., 2015, 21, 11773.

2 (a) F. Li, J. Nie, L. Sun, Y. Zheng and J.-A. Ma, Angew. Chem., Int. Ed., 2013, 52, 6255; (b) J. Wang, W.-G. Kong, F. Li, J. Liu, Q. Shen, L. Liu and W.-X. Zhao, Org. Biomol. Chem., 2015, 13, 5399; (c) Y. Zhu, Z. Dong, X. Cheng, X. Zhong, X. Liu, L. Lin, Z. Shen, P. Yang, Y. Li, H. Wang, W. Yan, K. Wang and R. Wang, Org. Lett., 2016, 18, 3546.

3 D. A. Evans and J. A. Gauchet-Prunet, J. Org. Chem., 1993, 58, 2446.

4 For a few selected examples, see: (a) D. T. Hung, J. B. Nerenberg and S. L. Schreiber, J. Am. Chem. Soc., 1996, 118, 11054; (b) T. J. Hunter and G. A. O'Doherty, Org. Lett., 2001, 3, 2777; (c) T. A. Dineen and W. R. Roush, Org. Lett., 2004, 6, 2043; (d) A. Vincent and J. Prunet, Synlett, 2006, 2269; (e) E. de Lemos, F.-H. Porée, A. Bourin, J. Barbion, E. Agouridas, M.-I. Lannou, A. Commerçon, J.-F. Betzer, A. Pancrazi and J. Ardisson, Chem. - Eur. J., 2008, 14, 11092; $(f)$ S. S. Palimkar and J. Uenishi, Org. Lett., 2010, 12, 4160; (g) A. M. M. Albury and M. P. Jennings, J. Org. Chem., 2012, 77, 6929; (h) R. W. Bates and T. G. Lek, Synthesis, 2014, 1731; (i) T. J. Hunter, Y. Wang, J. Zheng and G. A. O'Doherty, Synthesis, 2016, 1700.

5 D. A. Evans, P. Nagorny, D. J. Reynolds and K. J. McRae, Angew. Chem., Int. Ed., 2007, 46, 541.

6 (a) R. Oriez and J. Prunet, Tetrahedron Lett., 2010, 51, 256; (b) L. Grimaud, D. Rotulo, R. Ros-Perez, L. Guitry-Azam and J. Prunet, Tetrahedron Lett., 2002, 43, 7477.

7 D. Gamba-Sanchez and J. Prunet, J. Org. Chem., 2010, 75, 3129. 
8 (a) L. Grimaud, R. de Mesmay and J. Prunet, Org. Lett., 2002, 4, 419; (b) D. Rotulo-Sims and J. Prunet, Org. Lett., 2007, 9, 4147; (c) R. Aouzal and J. Prunet, Org. Biomol. Chem., 2009, 7, 3594; (d) R. Oriez, Master Thesis, Ecole Polytechnique (Palaiseau), 2006.

9 F. Li, J. Wang, M. Xu, X. Zhao, X. Zhou, W.-X. Zhao and L. Liu, Org. Biomol. Chem., 2016, 14, 3981.
10 J. A. Gazaille and T. Sammakia, Org. Lett., 2012, 14, 2678.

11 In our previous studies the typical base employed was $t \mathrm{BuOK}$ and we performed successive additions of a base and carbonyl compound.

12 A. Chattopadhyay, J. Org. Chem., 1996, 61, 6104.

13 P. A. Alexander, S. P. Marsden, D. M. Muñoz Subtil and J. C. Reader, Org. Lett., 2005, 7, 5433. 